\title{
Free Radical Binding
}

National Cancer Institute

\section{Source}

National Cancer Institute. Free Radical Binding. NCI Thesaurus. Code C40953.

Free Radical Binding involves temporary interaction with chemically unstable and highly reactive molecules (free radicals) having an unsatisfied electron valence pair. Agents of tissue damage through exposure to radiation, environment chemicals, and aging, reactive free radicals stabilize themselves by appropriating an electron from a nearby molecule and damaging that molecule by altering the electron number in its outermost electron shell. 